\title{
CONVERGENCE ON THE LEVI-CIVITA FIELD AND STUDY OF POWER SERIES
}

\author{
KHODR SHAMSEDDINE AND MARTIN BERZ
}

\begin{abstract}
Convergence under various topologies and analytical properties of power series on Levi-Civita fields are studied. A radius of convergence is established that asserts convergence under a weak topology and reduces to the conventional radius of convergence for real power series. It also asserts strong (order) convergence for points the distance of which from the center is infinitely smaller than the radius of convergence.

In addition to allowing the introduction of common transcendental functions, power series are shown to behave similar to real power series. Besides being infinitely often differentiable and re-expandable around other points, it is shown that power series satisfy a general intermediate value theorem as well as a maximum theorem and a mean value theorem.
\end{abstract}

\section{IntRoduction}

It is a known fact that topological continuity or differentiability of a function on a closed interval of any non-Archimedean field are not sufficient to guarantee that the function assumes all the intermediate values nor a maximum nor a minimum on the interval. These problems are common to all non-Archimedean sructures and are due to the total disconnectedness of these structures in the order topology. It

1991 Mathematics Subject Classification. 26E30, 12J25.

Key words and phrases. Levi-Civita field, power series, weak convergence, strong convergence, calculus.

This research was supported by an Alfred P. Sloan fellowship and by the Untied States Department of Energy, Grant \# DE-FG02-95ER40931. 
is shown in [1] that under stronger definitions for continuity and differentiability and under mild conditions, the intermediate value theorem can be proven to hold for functions on finite-length intervals of the Levi-Civita field $\mathcal{R}$.

An alternative solution to the difficulties mentioned above is to try to extend the real calculus to a large class of functions on the Levi-Civita field $\mathcal{R}$, discussed in [1]. It turns out that power series on $\mathcal{R}$ have all the nice smoothness properties that real power series have in $\mathbb{R}$. In particular, they are infinitely often differentiable; they are re-expandable around any point in their domain of convergence; and they satisfy all the common theorems of real calculus.

Previous work on power series on the Levi-Civita field has been restricted to power series with real coefficients. In $[5,6,7,4]$, they were studied for infinitely small arguments, while in $[2,3]$, using the weak topology, also finite arguments were possible. In this paper, we study the general case when the coefficients are LeviCivita numbers. We will focus here on the convergence criteria for power series and refer the reader to [10] for the details on their analytical properties. After reviewing two concepts of convergence: strong convergence and weak convergence [3], we show some new results concerning the convergence of sums and products of infinite series. Then we enhance and prove convergence criteria for power series, first stated in [2]. In particular, we derive a radius of weak convergence $\eta$ such that the power series converges weakly for all points the distance of which from the center is finitely smaller than $\eta$ and it converges strongly for all points the distance of which from the center is infinitely smaller than $\eta$.

Using the convergence properties of power series, we extend all the real transcendental functions to $\mathcal{R}$; this allows for the study of differentiability of real functions repesentable on a computer and for the computation of existing derivatives to high orders up to machine precision [9].

We start with a review of some basic and useful terminology and refer the reader to $[3,8]$ for a more detailed study of the field.

Definition 1.1 (The set $\mathcal{R}$ ). We define

$$
\mathcal{R}=\{f: \mathbb{Q} \rightarrow \mathbb{R} \mid\{x \mid f(x) \neq 0\} \text { is left-finite }\} .
$$

So the elements of $\mathcal{R}$ are those real valued functions on $\mathbb{Q}$ that are non-zero only on a left-finite set, i.e. below every rational number $q$ there are only finitely many points where the given functions do not vanish.

We denote elements of $\mathcal{R}$ by $x, y$, etc. and identify their values at $q \in \mathbb{Q}$ with brackets like $x[q]$. This avoids confusion when we consider functions on $\mathcal{R}$.

For the further discussion, it is convenient to introduce the following terminology:

Definition $1.2\left(\operatorname{supp}, \lambda, \sim, \approx,=_{r}\right)$. For $x \in \mathcal{R}$, we define $\operatorname{supp}(x)=\{q \in \mathbb{Q} \mid x[q] \neq 0\}$ and call it the support of $x$ $\lambda(x)=\min (\operatorname{supp}(x))$ for $x \neq 0$ (which exists because of left-finiteness) and $\lambda(0)=+\infty$.

Comparing two elements $x$ and $y$ in $\mathcal{R}$, we say

$x \sim y$ if and only if $\lambda(x)=\lambda(y)$

$x \approx y$ if and only if $\lambda(x)=\lambda(y)$ and $x[\lambda(x)]=y[\lambda(y)]$;

$x={ }_{r} y$ if and only if $x[q]=y[q]$ for all $q \leq r$.

At this point, these definitions may feel somewhat arbitrary; but after having introduced the concept of ordering on $\mathcal{R}$, we will see that $\lambda$ describes "orders 
of infinite largeness or smallness", the relation " $\approx "$ corresponds to agreement up to infinitely small relative error, while " " corresponds to agreement of order of magnitude.

Definition 1.3 (Addition and Multiplication on $\mathcal{R}$ ). We define addition on $\mathcal{R}$ componentwise: $(x+y)[q]=x[q]+y[q]$. Multiplication is defined as follows: For $q \in Q$ we set

$$
(x \cdot y)[q]=\sum_{\substack{q_{x}, q_{y} \in Q, q_{x}+q_{y}=q}} x\left[q_{x}\right] \cdot y\left[q_{y}\right] .
$$

Since elements of $\mathcal{R}$ have left-finite supports, only finitely many terms contribute to the sum in Equation (1.1). Thus, - is a well defined operation on $\mathcal{R}$. It turns out that the operations + and $\cdot$ make $(\mathcal{R},+, \cdot)$ into a field, in which we can isomorphically embed the reals as a subfield via the map $\Pi: R \rightarrow \mathcal{R}$ defined by

$$
\Pi(x)[q]=\left\{\begin{array}{ll}
x & \text { if } q=0 \\
0 & \text { else }
\end{array} .\right.
$$

Definition 1.4 (Ordering in $\mathcal{R}$ ). Let $x, y$ be distinct elements of $\mathcal{R}$. We say $x>y$ if and only if $(x-y)[\lambda(x-y)]>0$. Furthermore, we say $x<y$ if and only if $y>x$.

With this definition of the order relation, $\mathcal{R}$ is a totally ordered field. Moreover, the embedding $\Pi$ in (1.2) is compatible with the order.

Besides the usual order relations, some other notations are convenient:

Definition 1.5. ( $\gg, \gg)$ Let $a, b$ be non-negative. We say $a$ is infinitely smaller than $b$ (and write $a \ll b$ ) if and only if $n \cdot a<b$ for all natural $n$; we say $a$ is infinitely larger than $b$ (and write $a \gg b$ ) if and only if $b \ll a$. If $a \ll 1$, we say $a$ is infinitely small; if $1 \ll a$, we say $a$ is infinitely large. Infinitely small numbers are also called infinitesimals or differentials. Infinitely large numbers are also called infinite. Non-negative numbers that are neither infinitely small nor infinitely large are also called finite.

Definition 1.6 (The Number $d$ ). Let $d$ be the element of $\mathcal{R}$ given by $d[1]=1$ and $d[q]=0$ for $q \neq 1$.

It is easy to check that $0<d^{q} \ll 1$ if and only if $q>0$ and $d^{q} \gg 1$ if and only if $q<0$. It follows that, altogether, the Levi-Civita $\mathcal{R}$ is a totally ordered non-Archimedean field extension of the real numbers.

\section{Strong Convergence and Weak Convergence}

2.1. Strong Convergence. We begin this section by reviewing a special property of sequences.

Definition 2.1 (Regularity). A sequence $\left(s_{n}\right)$ in $\mathcal{R}$ is called regular if and only if the union of the supports of all members of the sequence is a left-finite subset of $\mathbb{Q}$.

This property is not automatically assured, as becomes apparent from considering the sequence $\left(d^{-n}\right)$. It is shown [3] that the property of regularity is compatible with the common operations of sequences. 
Definition 2.2 (Strong Convergence). Let $\left(s_{n}\right)$ be a sequence in $\mathcal{R}$. We say that $\left(s_{n}\right)$ is strongly convergent to the limit $s \in \mathcal{R}$ if and only if for every $\epsilon>0$ in $\mathcal{R}$ there exists $N \in \mathbb{N}$ such that $\left|s_{n}-s\right|<\epsilon$ for all $n \geq N$.

Like $\mathbb{R}$, the field $\mathcal{R}$ is Cauchy complete with respect to the order topology. That is, a sequence $\left(s_{n}\right)$ in $\mathcal{R}$ converges strongly if and only if $\left(s_{n}\right)$ is strongly Cauchy.

It is shown that every strongly convergent sequence in $\mathcal{R}$ is regular; moreover, we have the following useful convergence criterion the proof of which can be found in $[3]$.

Theorem 2.3 (Strong Convergence Criterion for sequences). Let $\left(s_{n}\right)$ be a sequence in $\mathcal{R}$. Then $\left(s_{n}\right)$ converges strongly in $\mathcal{R}$ if and only if for all $r \in \mathbb{Q}$ there exists $N \in \mathbb{N}$ such that $s_{m}={ }_{r} s_{l}$ for all $m, l \geq N$.

The following results: Theorem 2.4, Corollary 2.5, Corollary 2.6 and Corollary 2.7 do not hold in $\mathbb{R}$; the non-Archimedicity of $\mathcal{R}$ is the key to their proofs.

Theorem 2.4. Let $\left(s_{n}\right)$ be a sequence in $\mathcal{R}$. Then $\left(s_{n}\right)$ is strongly Cauchy if and only if $\left(s_{n+1}-s_{n}\right)$ is a null sequence.

Proof. Let $\left(s_{n}\right)$ be a Cauchy sequence in $\mathcal{R}$, and let $\epsilon>0$ in $\mathcal{R}$ be given. Then there exists $N \in \mathbb{N}$ such that $\left|s_{l}-s_{m}\right|<\epsilon$ for all $l, m \geq N$. In particular, $\left|s_{m+1}-s_{m}\right|<\epsilon$ for all $m \geq N$. Hence, $\lim _{n \rightarrow \infty}\left(s_{n+1}-s_{n}\right)=0$.

Now assume that $\left(s_{n+1}-s_{n}\right)$ is a null sequence in $\mathcal{R}$, and let $\epsilon>0$ in $\mathcal{R}$ be given. Then there exists $N \in \mathbb{N}$ such that $\left|s_{m+1}-s_{m}\right|<d \epsilon$ for all $m \geq N$. Let $k, l \geq N$ be given. Without loss of generality, we may assume that $k>l$. Then we have that

$$
\begin{aligned}
\left|s_{k}-s_{l}\right| & =\left|s_{k}-s_{k-1}+s_{k-1}-s_{k-2}+\cdots+s_{l+1}-s_{l}\right| \\
& \leq\left|s_{k}-s_{k-1}\right|+\left|s_{k-1}-s_{k-2}\right|+\cdots+\left|s_{l+1}-s_{l}\right| \\
& <(k-l) d \epsilon<\epsilon
\end{aligned}
$$

since $(k-l) d$ is infinitely small. Thus, $\left(s_{n}\right)$ is strongly Cauchy in $\mathcal{R}$.

Corollary 2.5. Let $\left(s_{n}\right)$ be a sequence in $\mathcal{R}$. Then, $\left(s_{n}\right)$ converges strongly if and only if $\left(s_{n+1}-s_{n}\right)$ is a null sequence with respect to the order topology.

Corollary 2.6. The infinite series $\sum_{n=0}^{\infty} a_{n}$ converges strongly in $\mathcal{R}$ if and only if the sequence $\left(a_{n}\right)$ is a null sequence in $\mathcal{R}$.

Corollary 2.7. The series $\sum_{n=0}^{\infty} a_{n}$ converges strongly if and only if it converges absolutely strongly, that is if and only if $\sum_{n=0}^{\infty}\left|a_{n}\right|$ converges strongly.

Like in $\mathbb{R}$, it is easy to show [8] that the sum and product of two strongly convergent sequences converge strongly to the expected limits. Furthermore, the non-Archimedicity of $\mathcal{R}$ gives us a nice result in Theorem 2.8, which does not hold in $\mathbb{R}$ without the additional requirement that one of the series converge absolutely.

Theorem 2.8. Let $\sum_{n=0}^{\infty} a_{n}$ and $\sum_{n=0}^{\infty} b_{n}$ be two infinite series converging strongly in $\mathcal{R}$ to $a$ and $b$, respectively. Then, the series $\sum_{n=0}^{\infty} c_{n}$, where $c_{n}=\sum_{j=0}^{n} a_{j} b_{n-j}$, converges strongly to $a \cdot b$ in $\mathcal{R}$.

Proof. First, we show that $\sum_{n=0}^{\infty} c_{n}$ converges strongly in $\mathcal{R}$. By Corollary 2.6, it suffices to show that $\lim _{n \rightarrow \infty} c_{n}=0$. Since $\sum_{n=0}^{\infty} a_{n}$ and $\sum_{n=0}^{\infty} b_{n}$ converge strongly in $\mathcal{R}$, the sequences $\left(a_{n}\right)$ and $\left(b_{n}\right)$ are both strongly null in $\mathcal{R}$. Hence $\left(a_{n}\right)$ 
and $\left(b_{n}\right)$ are both bounded. Therefore, there exists $B>0$ in $\mathcal{R}$ such that $\left|a_{n}\right|<B$ and $\left|b_{n}\right|<B$ for all $n \geq 0$. Let $\epsilon>0$ in $\mathcal{R}$ be given. Then, there exists $M \in \mathbb{N}$ such that $\left|a_{m}\right|<d \epsilon / B$ and $\left|b_{m}\right|<d \epsilon / B$ for all $m \geq M$. Let $N=2 M$. Then, for all $m \geq N$, we have that

$$
\begin{aligned}
\left|c_{m}\right| & =\left|a_{0} b_{m}+a_{1} b_{m-1}+\cdots+a_{m-1} b_{1}+a_{m} b_{0}\right| \\
& \leq\left|a_{0} b_{m}\right|+\left|a_{1} b_{m-1}\right|+\cdots+\left|a_{m-1} b_{1}\right|+\left|a_{m} b_{0}\right| \\
& =\left|a_{0}\right|\left|b_{m}\right|+\left|a_{1}\right|\left|b_{m-1}\right|+\cdots+\left|a_{m-1}\right|\left|b_{1}\right|+\left|a_{m}\right|\left|b_{0}\right| \\
& <B \frac{d \epsilon}{B}+B \frac{d \epsilon}{B}+\cdots+\frac{d \epsilon}{B} B+\frac{d \epsilon}{B} B \\
& =(m+1) d \epsilon<\epsilon .
\end{aligned}
$$

So, for all $\epsilon>0$ in $\mathcal{R}$, we can find $N \in \mathbb{N}$ such that $\left|c_{m}\right|<\epsilon$ for all $m \geq N$. Hence, $\lim _{n \rightarrow \infty} c_{n}=0$ and thus $\sum_{n=0}^{\infty} c_{n}$ converges strongly in $\mathcal{R}$. It remains to show that $\sum_{n=0}^{\infty} c_{n}=a \cdot b$.

Consider the sequence of partial sums $\left(s_{2 n}\right)$, where

$$
\begin{aligned}
s_{2 n}= & c_{0}+c_{1}+\cdots+c_{2 n}=\sum_{i+j=0}^{2 n} a_{i} b_{j} \\
= & \left(a_{0}+a_{1}+\cdots+a_{n}\right)\left(b_{0}+b_{1}+\cdots+b_{n}\right) \\
& +a_{0}\left(b_{n+1}+\cdots+b_{2 n}\right)+a_{1}\left(b_{n+1}+\cdots+b_{2 n-1}\right)+\cdots+a_{n-1} b_{n+1} \\
& +b_{0}\left(a_{n+1}+\cdots+a_{2 n}\right)+b_{1}\left(a_{n+1}+\cdots+a_{2 n-1}\right)+\cdots+b_{n-1} a_{n+1} .
\end{aligned}
$$

Note that

$$
\begin{aligned}
& \left|a_{0}\left(b_{n+1}+\cdots+b_{2 n}\right)+a_{1}\left(b_{n+1}+\cdots+b_{2 n-1}\right)+\cdots+a_{n-1} b_{n+1}\right| \\
\leq & \left|a_{0}\right|\left|b_{n+1}+\cdots+b_{2 n}\right|+\left|a_{1}\right|\left|b_{n+1}+\cdots+b_{2 n-1}\right|+\cdots+\left|a_{n-1}\right|\left|b_{n+1}\right| \\
\leq & B\left(\left|b_{n+1}+\cdots+b_{2 n}\right|+\left|b_{n+1}+\cdots+b_{2 n-1}\right|+\cdots+\left|b_{n+1}\right|\right) .
\end{aligned}
$$

Let $\epsilon>0$ in $\mathcal{R}$ be given. Since $\left(b_{n}\right)$ is a null sequence, there exists $N \in \mathbb{N}$ such that $\left|b_{n}\right|<d \epsilon / B$ for all $n \geq N$. Hence, for all $n \geq N$ and for all $p \in \mathbb{N}$, we have that

$$
\begin{aligned}
\left|b_{n+1}+\cdots+b_{n+p}\right| & \leq\left|b_{n+1}\right|+\cdots+\left|b_{n+p}\right| \\
& <\frac{d \epsilon}{B}+\cdots+\frac{d \epsilon}{B}=(p d) \cdot \frac{\epsilon}{B}=(\text { pnd }) \frac{\epsilon}{n B} \\
& <\frac{\epsilon}{n B},
\end{aligned}
$$

where, in the last step, we made use of the fact that $d$ is infinitely small and $p n$ is an integer, so that $p n d<1$. Therefore, for all $n \geq N$, we have that

$$
\begin{aligned}
& \left|a_{0}\left(b_{n+1}+\cdots+b_{2 n}\right)+a_{1}\left(b_{n+1}+\cdots+b_{2 n-1}\right)+\cdots+a_{n-1} b_{n+1}\right| \\
& <B(\underbrace{\frac{\epsilon}{n B}+\frac{\epsilon}{n B}+\cdots+\frac{\epsilon}{n B}}_{n \text { times }})=\epsilon .
\end{aligned}
$$

Therefore,

$$
\lim _{n \rightarrow \infty}\left(a_{0}\left(b_{n+1}+\cdots+b_{2 n}\right)+a_{1}\left(b_{n+1}+\cdots+b_{2 n-1}\right)+\cdots+a_{n-1} b_{n+1}\right)=0 .
$$

Similarly, we can show that

$$
\lim _{n \rightarrow \infty}\left(b_{0}\left(a_{n+1}+\cdots+a_{2 n}\right)+b_{1}\left(a_{n+1}+\cdots+a_{2 n-1}\right)+\cdots+b_{n-1} a_{n+1}\right)=0 .
$$


Hence

$$
\begin{aligned}
\lim _{n \rightarrow \infty} s_{2 n}= & \lim _{n \rightarrow \infty}\left(\left(a_{0}+a_{1}+\cdots+a_{n}\right)\left(b_{0}+b_{1}+\cdots+b_{n}\right)\right)+ \\
& \lim _{n \rightarrow \infty}\left(a_{0}\left(b_{n+1}+\cdots+b_{2 n}\right)+a_{1}\left(b_{n+1}+\cdots+b_{2 n-1}\right)+\cdots+a_{n-1} b_{n+1}\right)+ \\
& \lim _{n \rightarrow \infty}\left(b_{0}\left(a_{n+1}+\cdots+a_{2 n}\right)+b_{1}\left(a_{n+1}+\cdots+a_{2 n-1}\right)+\cdots+b_{n-1} a_{n+1}\right) \\
= & \lim _{n \rightarrow \infty}\left(\left(a_{0}+a_{1}+\cdots+a_{n}\right)\left(b_{0}+b_{1}+\cdots+b_{n}\right)\right) .
\end{aligned}
$$

Let $\left(A_{n}\right)$ and $\left(B_{n}\right)$ denote the sequences of partial sums of $\sum_{n=0}^{\infty} a_{n}$ and $\sum_{n=0}^{\infty} b_{n}$, respectively. Then $A_{n}=a_{0}+a_{1}+\cdots+a_{n}, \lim _{n \rightarrow \infty} A_{n}=a ; B_{n}=b_{0}+b_{1}+\cdots+b_{n}$, and $\lim _{n \rightarrow \infty} B_{n}=b$. Therefore,

$$
\lim _{n \rightarrow \infty} s_{2 n}=\lim _{n \rightarrow \infty}\left(A_{n} B_{n}\right)=\left(\lim _{n \rightarrow \infty} A_{n}\right)\left(\lim _{n \rightarrow \infty} B_{n}\right)=a \cdot b .
$$

Since $\sum_{n=0}^{\infty} c_{n}$ converges strongly, it has one and only one limit. Hence $\lim _{n \rightarrow \infty} s_{2 n}=$ $\lim _{n \rightarrow \infty} s_{2 n+1}=a \cdot b=\lim _{n \rightarrow \infty} s_{n} ;$ so

$$
\sum_{n=0}^{\infty} c_{n}=a \cdot b=\left(\sum_{n=0}^{\infty} a_{n}\right)\left(\sum_{n=0}^{\infty} b_{n}\right) .
$$

The following lemma is a consequence of the fact that the topology induced on $\mathbb{R}$ by the order topology in $\mathcal{R}$ is the discrete topology in $\mathbb{R}[3]$.

Lemma 2.9. Let $\left(s_{n}\right)$ be a sequence in $\mathcal{R}$ the members of which are purely real. Then $\left(s_{n}\right)$ converges strongly if and only if there exists $j \in \mathbb{N}$ such that $s_{m}=s_{j}$ for all $m \geq j$.

As we see, the concept of strong convergence provides very nice properties, and moreover strong convergence can be checked easily by virtue of Theorem 2.3 and Corollary 2.6. However, for some applications it is not sufficient, and it is advantageous to study a new kind of convergence.

2.2. Weak Convergence. We start this section by defining a family of semi-norms on $\mathcal{R}$, which induces on $\mathcal{R}$ a topology weaker than the order topology and called weak topology [3].

Definition 2.10. Given $r \in \mathbb{Q}$, we define a mapping $\|\cdot\|_{r}: \mathcal{R} \rightarrow \mathbb{R}$ as follows.

$$
\|x\|_{r}=\sup \{|x[q]|: q \leq r\} .
$$

Remark 2.11. The supremum in Equation (2.1) is finite and it is even a maximum since, for any $r$, only finitely many of the $x[q]$ 's considered do not vanish.

Definition 2.12. A sequence $\left(s_{n}\right)$ in $\mathcal{R}$ is said to be weakly convergent if and only if there exists $s \in \mathcal{R}$, called the weak limit of the sequence $\left(s_{n}\right)$, such that the sequence $\left(\left\|s_{n}-s\right\|_{r}\right)$ converges to 0 in $\mathbb{R}$ for all $r \in \mathbb{Q}$.

One immediately concludes that if the weak limit exists, then it is unique.

A detailed study of the properties of weak convergence is found in $[3,8]$. Here we will give only the following three results which will prove useful for studying convergence of power series in Section 3. For the proof of Theorem 2.13, we refer the reader to $[3]$. 
Theorem 2.13 (Convergence Criterion for Weak Convergence). Let the sequence $\left(s_{n}\right)$ converge weakly to the limit $s$. Then, the sequence $\left(s_{n}[q]\right)$ converges to $s[q]$ in $\mathbb{R}$, for all $q \in \mathbb{Q}$, and the convergence is uniform on every subset of $\mathbb{Q}$ bounded above. Let on the other hand $\left(s_{n}\right)$ be regular, and let the sequence $\left(s_{n}[q]\right)$ converge in $\mathbb{R}$ to $s[q]$ for all $q \in \mathbb{Q}$. Then $\left(s_{n}\right)$ converges weakly in $\mathcal{R}$ to $s$.

Theorem 2.14. Let $\left(s_{n}\right)$ and $\left(t_{n}\right)$ be two regular sequences in $\mathcal{R}$ converging weakly to $s$ and $t$, respectively. Then the sequence $\left(s_{n} t_{n}\right)$ converges weakly to $s \cdot t$.

Proof. Since $\left(s_{n}\right)$ and $\left(t_{n}\right)$ are both regular, so is $\left(s_{n} t_{n}\right)$. To show that $\left(s_{n} t_{n}\right)$ converges weakly to $s \cdot t$, it remains to show that the sequence $\left(\left(s_{n} t_{n}\right)[q]\right)$ converges in $\mathbb{R}$ to $(s \cdot t)[q]$ for all $q \in \mathbb{Q}$, using Theorem 2.13. Let $A=\cup_{n=0}^{\infty} \operatorname{supp}\left(a_{n}\right)$ and $B=\cup_{n=0}^{\infty} \operatorname{supp}\left(b_{n}\right)$. Then $A, B \in \mathcal{F}$. Let $q \in \mathbb{Q}$ be given. Then, for all $n$, we have that

$$
\left(s_{n} t_{n}\right)[q]=\sum_{\substack{q_{1}+q_{2}=q \\ q_{1} \in A, q_{2} \in B}} s_{n}\left[q_{1}\right] t_{n}\left[q_{2}\right]
$$

Since $A$ and $B$ are left-finite, only finitely many terms contribute to the sum in Equation (2.2); and we have that

$$
\begin{aligned}
\lim _{n \rightarrow \infty}\left(s_{n} t_{n}\right)[q] & =\lim _{n \rightarrow \infty}\left(\sum_{\substack{q_{1}+q_{2}=q \\
q_{1} \in A, q_{2} \in B}} s_{n}\left[q_{1}\right] t_{n}\left[q_{2}\right]\right) \\
& =\sum_{\substack{q_{1}+q_{2}=q \\
q_{1} \in A, q_{2} \in B}}\left(\lim _{n \rightarrow \infty}\left(s_{n}\left[q_{1}\right] t_{n}\left[q_{2}\right]\right)\right) \\
& =\sum_{\substack{q_{1}+q_{2}=q \\
q_{1} \in A, q_{2} \in B}}\left(\left(\lim _{n \rightarrow \infty} s_{n}\left[q_{1}\right]\right)\left(\lim _{n \rightarrow \infty} t_{n}\left[q_{2}\right]\right)\right) \\
& =\sum_{\substack{q_{1}+q_{2}=q \\
q_{1} \in A, q_{2} \in B}}\left(s\left[q_{1}\right] t\left[q_{2}\right]\right)=(s \cdot t)[q] .
\end{aligned}
$$

This finishes the proof of the theorem.

Theorem 2.15. If the series $\sum_{n=0}^{\infty} a_{n}$ and $\sum_{n=0}^{\infty} b_{n}$ are regular, $\sum_{n=0}^{\infty} a_{n}$ converges absolutely weakly to $a$, and $\sum_{n=0}^{\infty} b_{n}$ converges weakly to $b$, then $\sum_{n=0}^{\infty} c_{n}$, where $c_{n}=\sum_{j=0}^{n} a_{j} b_{n-j}$, converges weakly to $a \cdot b$.

Proof. Let $\left(A_{n}\right),\left(B_{n}\right)$, and $\left(C_{n}\right)$ be the sequences of partial sums of $\sum_{n=0}^{\infty} a_{n}$, $\sum_{n=0}^{\infty} b_{n}$, and $\sum_{n=0}^{\infty} c_{n}$, respectively. Then $\left(A_{n}\right)$ and $\left(B_{n}\right)$ are both regular, $\left(A_{n}\right)$ converges absolutely weakly to $a$ and $\left(B_{n}\right)$ converges weakly to $b$. Since $\left(A_{n}\right)$ and $\left(B_{n}\right)$ are both regular, so is $\left(C_{n}\right)$. It remains to show that $\left(C_{n}[q]\right)$ converges in $\mathbb{R}$ to $(a \cdot b)[q]$ for all $q \in \mathbb{Q}$.

Since $\left(A_{n}\right)$ converges absolutely weakly to $a,\left(A_{n}[t]\right)$ converges absolutely in $\mathbb{R}$ to $a[t]$ for all $t \in \mathbb{Q}$. Similarly, $\left(B_{n}[t]\right)$ converges in $\mathbb{R}$ to $b[t]$ for all $t \in \mathbb{Q}$. Let 
$A=\cup_{n=0}^{\infty} \operatorname{supp}\left(a_{n}\right)$ and $B=\cup_{n=0}^{\infty} \operatorname{supp}\left(b_{n}\right)$, and let $q \in \mathbb{Q}$ be given. Then

$$
\begin{aligned}
C_{n}[q] & =\left(\sum_{m=0}^{n} c_{m}\right)[q]=\sum_{m=0}^{n} c_{m}[q]=\sum_{m=0}^{n}\left(\left(\sum_{j=0}^{m} a_{j} b_{m-j}\right)[q]\right) \\
& =\sum_{m=0}^{n}\left(\sum_{j=0}^{m}\left(a_{j} b_{m-j}\right)[q]\right)=\sum_{m=0}^{n} \sum_{j=0}^{m}\left(\sum_{\substack{q_{1}+q_{2}=q \\
q_{1} \in A, q_{2} \in B}} a_{j}\left[q_{1}\right] b_{m-j}\left[q_{2}\right]\right) \\
& =\sum_{\substack{q_{1}+q_{2}=q \\
q_{1} \in A, q_{2} \in B}}\left(\sum_{m=0}^{n} \sum_{j=0}^{m} a_{j}\left[q_{1}\right] b_{m-j}\left[q_{2}\right]\right) \text { because of regularity } \\
& =\sum_{\substack{q_{1}+q_{2}=q \\
q_{1} \in A, q_{2} \in B}}\left(\sum_{m=0}^{n}\left(\sum_{j=0}^{m} a_{j}\left[q_{1}\right] b_{m-j}\left[q_{2}\right]\right)\right) .
\end{aligned}
$$

Since $\sum_{n=0}^{\infty} a_{n}\left[q_{1}\right]$ converges absolutely to $a\left[q_{1}\right]$ and since $\sum_{n=0}^{\infty} b_{n}\left[q_{2}\right]$ converges to $b\left[q_{2}\right]$, we have that $\lim _{n \rightarrow \infty}\left(\sum_{m=0}^{n}\left(\sum_{j=0}^{m} a_{j}\left[q_{1}\right] b_{m-j}\left[q_{2}\right]\right)\right)$ exists in $\mathbb{R}$ and is equal to $a\left[q_{1}\right] b\left[q_{2}\right]$. Since the sum over the $q$ 's is finite because of left-finiteness of $A$ and $B$, we have also that

$$
\lim _{n \rightarrow \infty}\left(\sum_{\substack{q_{1}+q_{2}=q \\ q_{1} \in A, q_{2} \in B}}\left(\sum_{m=0}^{n}\left(\sum_{j=0}^{m} a_{j}\left[q_{1}\right] b_{m-j}\left[q_{2}\right]\right)\right)\right)
$$

exists in $\mathbb{R}$ and is equal to

$$
\sum_{\substack{q_{1}+q_{2}=q \\ q_{1} \in A, q_{2} \in B}}\left(\lim _{n \rightarrow \infty}\left(\sum_{m=0}^{n}\left(\sum_{j=0}^{m} a_{j}\left[q_{1}\right] b_{m-j}\left[q_{2}\right]\right)\right) .\right.
$$

Hence $\lim _{n \rightarrow \infty} C_{n}[q]$ exists in $\mathbb{R}$ and we have that

$$
\lim _{n \rightarrow \infty} C_{n}[q]=\sum_{\substack{q_{1}+q_{2}=q \\ q_{1} \in A, q_{2} \in B}} a\left[q_{1}\right] b\left[q_{2}\right]=(a \cdot b)[q] .
$$

Since $\left(C_{n}\right)$ is regular and since $\lim _{n \rightarrow \infty} C_{n}[q]=(a \cdot b)[q]$ for all $q \in \mathbb{Q},\left(C_{n}\right)$ converges weakly in $\mathcal{R}$ to $a \cdot b$. Therefore, $\sum_{n=0}^{\infty} c_{n}$ converges weakly to $a \cdot b$, and we can write

$$
\sum_{n=0}^{\infty} c_{n}=a \cdot b=\left(\sum_{n=0}^{\infty} a_{n}\right)\left(\sum_{n=0}^{\infty} b_{n}\right) .
$$

Finally, it is shown [3] that $\mathcal{R}$ is not Cauchy complete with respect to the weak topology and that strong convergence implies weak convergence to the same limit. 


\section{Power SERIES}

We now discuss a very important class of sequences, namely, the power series. We first study general criteria for power series to converge strongly or weakly. Once their convergence properties are established, they will allow the extension of many important real functions, and they will also provide the key for an exhaustive study of differentiability of all functions that can be represented on a computer (see [9]). Also based on our knowledge of the convergence properties of power series, we are able to study [10] a large class of functions on $\mathcal{R}$ that have all the nice smoothness properties that real power series have in $\mathbb{R}$. We begin our discussion of power series with an observation [3].

Lemma 3.1. Let $M \subset \mathbb{Q}$ be left-finite. Define

$$
M_{\Sigma}=\left\{q_{1}+\ldots+q_{n}: n \in \mathbb{N}, \text { and } q_{1}, \ldots, q_{n} \in M\right\}
$$

then $M_{\Sigma}$ is left-finite if and only if $\min (M) \geq 0$.

Corollary 3.2. The sequence $\left(x^{n}\right)$ is regular if and only if $\lambda(x) \geq 0$.

Let $\left(a_{n}\right)$ be a sequence in $\mathcal{R}$. Then the sequences $\left(a_{n} x^{n}\right)$ and $\left(\sum_{j=0}^{n} a_{j} x^{j}\right)$ are regular if $\left(a_{n}\right)$ is regular and $\lambda(x) \geq 0$.

\subsection{Convergence Criteria.}

Theorem 3.3 (Strong Convergence Criterion for Power Series). Let $\left(a_{n}\right)$ be a sequence in $\mathcal{R}$, and let

$$
\lambda_{0}=-\liminf _{n \rightarrow \infty}\left(\frac{\lambda\left(a_{n}\right)}{n}\right)=\limsup _{n \rightarrow \infty}\left(\frac{-\lambda\left(a_{n}\right)}{n}\right) \text { in } \mathbb{R} \cup\{-\infty, \infty\} .
$$

Let $x_{0} \in \mathcal{R}$ be fixed and let $x \in \mathcal{R}$ be given. Then the power series $\sum_{n=0}^{\infty} a_{n}\left(x-x_{0}\right)^{n}$ converges strongly in $\mathcal{R}$ if $\lambda\left(x-x_{0}\right)>\lambda_{0}$ and is strongly divergent if $\lambda\left(x-x_{0}\right)<\lambda_{0}$ or if $\lambda\left(x-x_{0}\right)=\lambda_{0}$ and $-\lambda\left(a_{n}\right) / n>\lambda_{0}$ for infinitely many $n$.

Proof. First assume that $\lambda\left(x-x_{0}\right)>\lambda_{0}$. To show that $\sum_{n=0}^{\infty} a_{n}\left(x-x_{0}\right)^{n}$ converges strongly in $\mathcal{R}$, it suffices, using Corollary 2.6 , to show that the sequence $\left(a_{n}\left(x-x_{0}\right)^{n}\right)$ is a null sequence with respect to the order topology. Since $\lambda\left(x-x_{0}\right)>\lambda_{0}$, there exists $t>0$ in $\mathbb{Q}$ such that $\lambda\left(x-x_{0}\right)-t>\lambda_{0}$. Hence there exists $N \in \mathbb{N}$ such that $\lambda\left(x-x_{0}\right)-t>-\lambda\left(a_{n}\right) / n$ for all $n \geq N$. Thus, $\lambda\left(a_{n}\left(x-x_{0}\right)^{n}\right)=\lambda\left(a_{n}\right)+n \lambda\left(x-x_{0}\right)>$ $n t$ for all $n \geq N$. Since $t>0$, we obtain that $\left(a_{n}\left(x-x_{0}\right)^{n}\right)$ is a null sequence with respect to the order topology.

Now assume that $\lambda\left(x-x_{0}\right)<\lambda_{0}$. To show that $\sum_{n=0}^{\infty} a_{n}\left(x-x_{0}\right)^{n}$ is strongly divergent in $\mathcal{R}$, it suffices to show that the sequence $\left(a_{n}\left(x-x_{0}\right)^{n}\right)$ is not a null sequence with respect to the order topology. Since $\lambda\left(x-x_{0}\right)<\lambda_{0}$, for all $N \in \mathbb{N}$ there exists $n>N$ such that $\lambda\left(x-x_{0}\right)<-\lambda\left(a_{n}\right) / n$. Hence, for all $N \in \mathbb{N}$, there exists $n>N$ such that $\lambda\left(a_{n}\left(x-x_{0}\right)^{n}\right)<0$, which entails that the sequence $\left(a_{n}\left(x-x_{0}\right)^{n}\right)$ is not a null sequence with respect to the order topology.

Finally, assume that $\lambda\left(x-x_{0}\right)=\lambda_{0}$ and $-\lambda\left(a_{n}\right) / n>\lambda_{0}$ for infinitely many $n$. Then for all $N \in \mathbb{N}$, there exists $n>N$ such that $-\lambda\left(a_{n}\right) / n>\lambda_{0}=\lambda\left(x-x_{0}\right)$. Thus, for each $N \in \mathbb{N}$, there exists $n>N$ such that $\lambda\left(a_{n}\left(x-x_{0}\right)^{n}\right)<0$. Therefore, the sequence $\left(a_{n}\left(x-x_{0}\right)^{n}\right)$ is not a null sequence with respect to the order topology; and hence $\sum_{n=0}^{\infty} a_{n}\left(x-x_{0}\right)^{n}$ is strongly divergent in $\mathcal{R}$.

The following two examples show that for the case when $\lambda\left(x-x_{0}\right)=\lambda_{0}$ and $-\lambda\left(a_{n}\right) / n \geq \lambda_{0}$ for only finitely many $n$, the series $\sum_{n=0}^{\infty} a_{n}\left(x-x_{0}\right)^{n}$ can either 
converge or diverge strongly. For this case, Theorem 3.7 provides a test for weak convergence.

Example 3.4. For each $n \geq 0$, let $a_{n}=d$; and let $x_{0}=0$ and $x=1$. Then $\lambda_{0}=$ $\limsup _{n \rightarrow \infty}(-1 / n)=0=\lambda(x)$. Moreover, we have that $-\lambda\left(a_{n}\right) / n=-1 / n<\lambda_{0}$ for all $n \geq 0$; and $\sum_{n=0}^{\infty} a_{n} x^{n}=\sum_{n=0}^{\infty} d$ is strongly divergent in $\mathcal{R}$.

Example 3.5. For each $n$, let $q_{n} \in \mathbb{Q}$ be such that $\sqrt{n} / 2<q_{n}<\sqrt{n}$, let $a_{n}=d^{q_{n}}$; and let $x_{0}=0$ and $x=1$. Then $\lambda_{0}=\limsup _{n \rightarrow \infty}\left(-q_{n} / n\right)=0=\lambda(x)$. Moreover, we have that $-\lambda\left(a_{n}\right) / n=-q_{n} / n<0=\lambda_{0}$ for all $n \geq 0$; and $\sum_{n=0}^{\infty} a_{n} x^{n}=$ $\sum_{n=0}^{\infty} d^{q_{n}}$ converges strongly in $\mathcal{R}$ since the sequence $\left(d^{q_{n}}\right)$ is a null sequence with respect to the order topology.

Remark 3.6. Let $x_{0}$ and $\lambda_{0}$ be as in Theorem 3.3, and let $x \in \mathcal{R}$ be such that $\lambda\left(x-x_{0}\right)=\lambda_{0}$. Then $\lambda_{0} \in \mathbb{Q} \cup\{\infty\}$. But if $\lambda_{0}=\infty$, then $x=x_{0}$ and hence $\sum_{n=0}^{\infty} a_{n}\left(x-x_{0}\right)^{n}=0$. So it remains to discuss the case when $\lambda\left(x-x_{0}\right)=\lambda_{0} \in \mathbb{Q}$.

Theorem 3.7 (Weak Convergence Criterion for Power Series). Let $\left(a_{n}\right)$ be a sequence in $\mathcal{R}$, and let $\lambda_{0}=\limsup _{n \rightarrow \infty}\left(-\lambda\left(a_{n}\right) / n\right) \in \mathbb{Q}$. Let $x_{0} \in \mathcal{R}$ be fixed, and let $x \in \mathcal{R}$ be such that $\lambda\left(x-x_{0}\right)=\lambda_{0}$. For each $n \geq 0$, let $b_{n}=a_{n} d^{n \lambda_{0}}$. Suppose that the sequence $\left(b_{n}\right)$ is regular and write $\cup_{n=0}^{\infty} \operatorname{supp}\left(b_{n}\right)=\left\{q_{1}, q_{2}, \ldots\right\}$; with $q_{j_{1}}<q_{j_{2}}$ if $j_{1}<j_{2}$. For each $n$, write $b_{n}=\sum_{j=1}^{\infty} b_{n_{j}} d^{q_{j}}$, where $b_{n_{j}}=b_{n}\left[q_{j}\right]$. Let $r=1 / \sup \left\{\limsup _{n \rightarrow \infty}\left|b_{n_{j}}\right|^{1 / n}: j \geq 1\right\}$. Then $\sum_{n=0}^{\infty} a_{n}\left(x-x_{0}\right)^{n}$ converges absolutely weakly in $\mathcal{R}$ if $\left|\left(x-x_{0}\right)\left[\lambda_{0}\right]\right|<r$ and is weakly divergent in $\mathcal{R}$ if $\left|\left(x-x_{0}\right)\left[\lambda_{0}\right]\right|>$ $r$.

Proof. Letting $y=d^{-\lambda_{0}}\left(x-x_{0}\right)$, we obtain that

$$
\lambda(y)=0=\limsup _{n \rightarrow \infty}\left(\frac{-\lambda\left(b_{n}\right)}{n}\right), \text { and } a_{n}\left(x-x_{0}\right)^{n}=b_{n} y^{n} \text { for all } n \geq 0 .
$$

So without loss of generality, we may assume that $x_{0}=0 ; \lambda_{0}=0=\lambda(x)$; and $b_{n}=a_{n}$ for all $n \geq 0$. Let $X=\Re(x)$; then $X \neq 0$. First assume that $|X|<r$.

First Claim: For all $j \geq 1$, we have that $\sum_{n=0}^{\infty} a_{n_{j}} X^{n}$ converges in $\mathbb{R}$.

Proof of the first claim: Since $|X|<r$, we have that

$$
\frac{1}{|X|}>\sup \left\{\limsup _{n \rightarrow \infty}\left|a_{n_{j}}\right|^{1 / n}: j \geq 1\right\}
$$

and hence $|X|<1 / \lim \sup _{n \rightarrow \infty}\left|a_{n_{j}}\right|^{1 / n}$ for all $j \geq 1$. Hence $\sum_{n=0}^{\infty} a_{n_{j}} X^{n}$ converges in $\mathbb{R}$ for all $j \geq 1$.

Second claim: For all $j \geq 1, \sum_{n=0}^{\infty} a_{n_{j}} x^{n}$ converges weakly in $\mathcal{R}$.

Proof of the second claim: Let $j \geq 1$ be given. For each $n$, let $A_{n_{j}}(x)=\sum_{i=0}^{n} a_{i_{j}} x^{i}$. So we need to show that the sequence $\left(A_{n_{j}}(x)\right)_{n \geq 0}$ is weakly convergent. Using Corollary 3.2, the sequence is regular since $\lambda(x) \geq 0$ and since the sequence $\left(a_{i_{j}}\right)$ is purely real and hence regular. Thus, it suffices to show that the sequence $\left(A_{n_{j}}(x)[t]\right)$ converges in $\mathbb{R}$ for all $t \in \mathbb{Q}$. Let $s=x-X$. If $s=0$, then we are done. So we may assume that $s \neq 0$. Let $t \in \mathbb{Q}$ be given; and choose $m \in \mathbb{N}$ such that $m \lambda(s)>t$. Then $(X+s)^{n}$ evaluated at $t$ yields:

$$
\left((X+s)^{n}\right)[t]=\left(\sum_{l=0}^{n} s^{l} \frac{n !}{(n-l) ! l !} X^{n-l}\right)[t]=\sum_{l=0}^{\min \{m, n\}} s^{l}[t] \frac{n !}{(n-l) ! l !} X^{n-l} .
$$


For the last equality, we used the fact that $s^{l}$ vanishes at $t$ for $l>m$. So we get the following chain of inequalities for any $\nu_{2}>\nu_{1}>m$ :

$$
\begin{aligned}
\sum_{n=\nu_{1}}^{\nu_{2}}\left|a_{n_{j}}(X+s)^{n}[t]\right| & =\sum_{n=\nu_{1}}^{\nu_{2}}\left|a_{n_{j}}\right|\left|\sum_{l=0}^{\min \{m, n\}} s^{l}[t] \frac{n !}{(n-l) ! l !} X^{n-l}\right| \\
& \leq \sum_{n=\nu_{1}}^{\nu_{2}} \sum_{l=0}^{m}\left|a_{n_{j}}\right|\left|s^{l}[t]\right| \frac{n !}{(n-l) ! l !}|X|^{n-l} \\
& \leq\left(\sum_{l=0}^{m} \frac{\left|s^{l}[t]\right||X|^{m-l}}{l !}\right)\left(\sum_{n=\nu_{1}}^{\nu_{2}}\left|a_{n_{j}}\right| n^{m}|X|^{n-m}\right) .
\end{aligned}
$$

Note that the right sum contains only real terms. As $|X|<r$, the series converges; the additional factor $n^{m}$ does not influence convergence since $\lim _{n \rightarrow \infty} \sqrt[n]{n^{m}}=1$. As the left hand term does not depend on $\nu_{1}$ and $\nu_{2}$, we therefore obtain absolute convergence at $t$. This finishes the proof of the second claim.

Third claim: $\sum_{n=0}^{\infty} a_{n} x^{n}$ converges weakly in $\mathcal{R}$.

Proof of the third claim: By the result of the second claim, we have that $\sum_{n=0}^{\infty} a_{n_{j}} x^{n}$ converges weakly in $\mathcal{R}$ for all $j \geq 1$. For each $j$, let $f_{j}(x)=\sum_{n=0}^{\infty} a_{n_{j}} x^{n}$; then $\lambda\left(f_{j}(x)\right) \geq 0$ for all $j \geq 1$. Thus $\sum_{j=1}^{\infty} d^{q_{j}} f_{j}(x)$ converges strongly (and hence weakly) in $\mathcal{R}$. Now let $t \in \mathbb{Q}$ be given. Then there exists $m \in \mathbb{N}$ such that $q_{j}>t$ for all $j \geq m$. Thus,

$$
\begin{aligned}
& \left(\sum_{j=1}^{\infty} d^{q_{j}} f_{j}(x)\right)[t]=\sum_{j=1}^{\infty}\left(d^{q_{j}} f_{j}(x)\right)[t]=\sum_{j=1}^{\infty}\left(\sum_{t_{1}+t_{2}=t} d^{q_{j}}\left[t_{1}\right] f_{j}(x)\left[t_{2}\right]\right) \\
= & \sum_{j=1}^{m}\left(\sum_{t_{1}+t_{2}=t} d^{q_{j}}\left[t_{1}\right] f_{j}(x)\left[t_{2}\right]\right)=\sum_{j=1}^{m} \sum_{t_{1}+t_{2}=t} d^{q_{j}}\left[t_{1}\right]\left(\sum_{n=0}^{\infty} a_{n_{j}} x^{n}\right)\left[t_{2}\right] \\
= & \sum_{j=1}^{m} \sum_{t_{1}+t_{2}=t} d^{q_{j}}\left[t_{1}\right] \sum_{n=0}^{\infty} a_{n_{j}} x^{n}\left[t_{2}\right]=\sum_{n=0}^{\infty} \sum_{j=1}^{m} a_{n_{j}}\left(\sum_{t_{1}+t_{2}=t} d^{q_{j}}\left[t_{1}\right] x^{n}\left[t_{2}\right]\right) \\
= & \sum_{n=0}^{\infty} \sum_{j=1}^{\infty} a_{n_{j}}\left(\sum_{t_{1}+t_{2}=t} d^{q_{j}}\left[t_{1}\right] x^{n}\left[t_{2}\right]\right)=\sum_{n=0}^{\infty} \sum_{j=1}^{\infty} a_{n_{j}}\left(d^{q_{j}} x^{n}\right)[t] \\
= & \left(\sum_{n=0}^{\infty} \sum_{j=1}^{\infty} a_{n_{j}} d^{q_{j}} x^{n}\right)[t]=\left(\sum_{n=0}^{\infty}\left(\sum_{j=1}^{\infty} a_{n_{j}} d^{q_{j}}\right) x^{n}\right)[t]=\left(\sum_{n=0}^{\infty} a_{n} x^{n}\right)[t] .
\end{aligned}
$$

This is true for all $t \in \mathbb{Q}$. Thus, $\sum_{n=0}^{\infty} a_{n} x^{n}$ converges weakly to $\sum_{j=1}^{\infty} d^{q_{j}} f_{j}(x)$.

Now assume that $|X|>r$. Then $1 /|X|<\sup \left\{\limsup _{n \rightarrow \infty}\left|a_{n_{j}}\right|^{1 / n}: j \geq 1\right\}$. Hence there exists $j_{0} \in \mathbb{N}$ such that $1 /|X|<\lim \sup _{n \rightarrow \infty}\left|a_{n_{j_{0}}}\right|^{1 / n}$. Thus, $|X|>$ $1 / \lim \sup _{n \rightarrow \infty}\left|a_{n_{j_{0}}}\right|^{1 / n}$; and hence $\sum_{n=0}^{\infty} a_{n_{j_{0}}} X^{n}$ diverges in $\mathbb{R}$. It follows that $\left(\sum_{n=0}^{\infty} a_{n} x^{n}\right)\left[q_{j_{0}}\right]$ diverges in $\mathbb{R}$; and hence $\sum_{n=0}^{\infty} a_{n} x^{n}$ is weakly divergent in $\mathcal{R}$.

The following two examples show that when the sequence $\left(b_{n}\right)$ in Theorem 3.7 is not regular, the power series may converge or diverge weakly for a given $x \in \mathcal{R}$ 
satisfying

$$
0<\left|\left(x-x_{0}\right)\left[\lambda_{0}\right]\right|<r=\frac{1}{\sup \left\{\limsup _{n \rightarrow \infty}\left|b_{n}[q]\right|^{1 / n}: q \in \mathbb{Q}\right\}} .
$$

Example 3.8. For each $n \geq 0$, let $a_{n}=d^{1 /(n+1)}$; then

$$
\begin{aligned}
\lambda_{0} & =\limsup _{n \rightarrow \infty}\left(\frac{-\lambda\left(a_{n}\right)}{n}\right)=0, \\
b_{n} & =a_{n} d^{n \lambda_{0}}=d^{1 /(n+1)} \text { for all } n \geq 0, \text { and } \\
r & =\frac{1}{\sup \left\{\lim \sup _{n \rightarrow \infty}\left|b_{n}[q]\right|^{1 / n}: q \in \mathbb{Q}\right\}}=\infty .
\end{aligned}
$$

We now show that even though $r=\infty$, the power series $\sum_{n=0}^{\infty} a_{n} x^{n}$ is weakly divergent for all real points $x \neq 0$. So let $x \neq 0$ be given in $\mathbb{R}$. Assume that $\sum_{n=0}^{\infty} a_{n} x^{n}$ converges weakly in $\mathcal{R}$ and let $s$ denote the weak limit. Then by Theorem 2.13, we have that

$$
s[q]=\sum_{n=0}^{\infty}\left(a_{n} x^{n}\right)[q] \text { for all } q \in \mathbb{Q} .
$$

Since $x \in \mathbb{R}$, we obtain that

$$
s[q]=\sum_{n=0}^{\infty} a_{n}[q] x^{n} \text { for all } q \in \mathbb{Q} .
$$

In particular, we obtain that

$$
s[1 /(m+1)]=\sum_{n=0}^{\infty} d^{1 /(n+1)}[1 /(m+1)] x^{n}=x^{m} \neq 0 \text { for all } m \in \mathbb{N} .
$$

This means that $1 /(m+1) \in \operatorname{supp}(s)$ for all $m \in \mathbb{N}$, which contradicts the fact that $s \in \mathcal{R}$ and that $\operatorname{supp}(s)$ is left-finite. Thus $\sum_{n=0}^{\infty} a_{n} x^{n}$ is weakly divergent.

Example 3.9. For each $k \in \mathbb{N}$, let

$$
a_{2 k-1}=-a_{2 k}=\frac{1}{2 k-1} d^{1 /(2 k-1)}
$$

Then

$$
\begin{aligned}
\lambda_{0} & =\limsup _{n \rightarrow \infty}\left(\frac{-\lambda\left(a_{n}\right)}{n}\right)=0, \\
b_{n} & =a_{n} d^{n \lambda_{0}}=a_{n} \text { for all } n \geq 0, \text { and } \\
r & =\frac{1}{\sup \left\{\lim \sup _{n \rightarrow \infty}\left|b_{n}[q]\right|^{1 / n}: q \in \mathbb{Q}\right\}}=\infty .
\end{aligned}
$$

Hence the sequence $\left(b_{n}\right)$ is not regular since $1 /(2 k-1) \in \cup_{n=0}^{\infty} \operatorname{supp}\left(b_{n}\right)$ for all $k \in \mathbb{N}$. We will show that $\sum_{n=0}^{\infty} a_{n} x^{n}$ converges weakly to 0 in $\mathcal{R}$ for $x=1$.

To show that $\sum_{n=0}^{\infty} a_{n}$ converges weakly to 0 , let $\epsilon>0$ be given in $\mathbb{R}$ and let $N \in \mathbb{N}$ be such that

$$
N>\max \left\{\epsilon, \frac{1}{\epsilon}\right\} \text {. }
$$

Then for all $m \geq N$, we have that

$$
\left\|\sum_{n=0}^{m} a_{n}\right\|_{1 / \epsilon} \leq \frac{1}{m} \leq \frac{1}{N}<\epsilon
$$


Thus, $\sum_{n=0}^{\infty} a_{n}$ converges weakly to 0 in $\mathcal{R}$. On the other hand, it is easy to check (using a similar argument as that in Example 3.8) that $\sum_{n=0}^{\infty} a_{n} x^{n}$ is weakly divergent in $\mathcal{R}$ for all $x \in \mathbb{R} \backslash\{0,1\}$.

Corollary 3.10 (Power Series with Purely Real Coefficients). Let $\sum_{n=0}^{\infty} a_{n} X^{n}$ be a power series with purely real coefficients and with classical radius of convergence equal to $\eta$. Let $x \in \mathcal{R}$, and let $A_{n}(x)=\sum_{i=0}^{n} a_{i} x^{i} \in \mathcal{R}$. Then, for $|x|<\eta$ and $|x| \not \approx \eta$, the sequence $\left(A_{n}(x)\right)$ converges absolutely weakly. We define the limit to be the continuation of the power series on $\mathcal{R}$.

Using Corollary 3.10, we can now extend real functions representable by power series to the Levi-Civita field $\mathcal{R}$.

Definition 3.11 (The Functions Exp, Cos, Sin, Cosh, and Sinh). By Corollary 3.10 , the series

$$
\sum_{n=0}^{\infty} \frac{x^{n}}{n !}, \sum_{n=0}^{\infty}(-1)^{n} \frac{x^{2 n}}{(2 n) !}, \sum_{n=0}^{\infty}(-1)^{n} \frac{x^{2 n+1}}{(2 n+1) !}, \sum_{n=0}^{\infty} \frac{x^{2 n}}{(2 n) !}, \text { and } \sum_{n=0}^{\infty} \frac{x^{2 n+1}}{(2 n+1) !}
$$

converge absolutely weakly in $\mathcal{R}$ for any $x \in \mathcal{R}$, at most finite in absolute value. For any such $x$, define

$$
\begin{aligned}
\exp (x) & =\sum_{n=0}^{\infty} \frac{x^{n}}{n !} \\
\cos (x) & =\sum_{n=0}^{\infty}(-1)^{n} \frac{x^{2 n}}{(2 n) !} \\
\sin (x) & =\sum_{n=0}^{\infty}(-1)^{n} \frac{x^{2 n+1}}{(2 n+1) !} \\
\cosh (x) & =\sum_{n=0}^{\infty} \frac{x^{2 n}}{(2 n) !} \\
\sinh (x) & =\sum_{n=0}^{\infty} \frac{x^{2 n+1}}{(2 n+1) !}
\end{aligned}
$$

A detailed study of the transcendental functions introduced on $\mathcal{R}$ in Definition 3.11 can be found in [8]. In particular, using Theorem 2.15 and Definition 3.11, we show that addition theorems similar to the real ones hold for these functions.

Theorem 3.12 (Addition Theorem). Let $x_{1}, x_{2} \in \mathcal{R}$ be at most finite in absolute value. Then

$$
\begin{aligned}
\exp \left(x_{1}+x_{2}\right) & =\exp \left(x_{1}\right) \exp \left(x_{2}\right) \\
\cos \left(x_{1} \pm x_{2}\right) & =\cos \left(x_{1}\right) \cos \left(x_{2}\right) \mp \sin \left(x_{1}\right) \sin \left(x_{2}\right), \\
\sin \left(x_{1} \pm x_{2}\right) & =\sin \left(x_{1}\right) \cos \left(x_{2}\right) \pm \cos \left(x_{1}\right) \sin \left(x_{2}\right), \\
\cosh \left(x_{1} \pm x_{2}\right) & =\cosh \left(x_{1}\right) \cosh \left(x_{2}\right) \pm \sinh \left(x_{1}\right) \sinh \left(x_{2}\right), \text { and } \\
\sinh \left(x_{1} \pm x_{2}\right) & =\sinh \left(x_{1}\right) \cosh \left(x_{2}\right) \pm \cosh \left(x_{1}\right) \sinh \left(x_{2}\right) .
\end{aligned}
$$

Proof. We will prove that Equation (3.1) is true; the rest follows similarly. Using Definition 3.11, we have that

$$
\exp \left(x_{1}\right)=\sum_{n=0}^{\infty} \frac{x_{1}^{n}}{n !} \text { and } \exp \left(x_{2}\right)=\sum_{n=0}^{\infty} \frac{x_{2}^{n}}{n !}
$$


where the series are both regular by Corollary 3.2, and they both converge absolutely weakly in $\mathcal{R}$. Thus, applying Theorem 2.15 , we obtain that $\sum_{n=0}^{\infty} c_{n}$ converges weakly in $\mathcal{R}$ and

$$
\left(\sum_{n=0}^{\infty} \frac{x_{1}^{n}}{n !}\right)\left(\sum_{n=0}^{\infty} \frac{x_{2}^{n}}{n !}\right)=\sum_{n=0}^{\infty} c_{n},
$$

where, for all $n \geq 0$,

$$
c_{n}=\sum_{j=0}^{n} \frac{x_{1}^{j}}{j !} \frac{x_{2}^{(n-j)}}{(n-j) !} .
$$

Hence,

$$
\begin{aligned}
\exp \left(x_{1}\right) \exp \left(x_{2}\right) & =\left(\sum_{n=0}^{\infty} \frac{x_{1}^{n}}{n !}\right)\left(\sum_{n=0}^{\infty} \frac{x_{2}^{n}}{n !}\right)=\sum_{n=0}^{\infty}\left(\sum_{j=0}^{n} \frac{x_{1}^{j}}{j !} \frac{x_{2}^{(n-j)}}{(n-j) !}\right) \\
& =\sum_{n=0}^{\infty} \frac{1}{n !}\left(\sum_{j=0}^{n} \frac{n !}{j !(n-j) !} x_{1}^{j} x_{2}^{(n-j)}\right)=\sum_{n=0}^{\infty} \frac{1}{n !}\left(x_{1}+x_{2}\right)^{n} \\
& =\exp \left(x_{1}+x_{2}\right) .
\end{aligned}
$$

3.2. Calculus. It turns out that power series on $\mathcal{R}$ behave similarly to real power series. It is shown [10] that within their radius of convergence, power series are infinitely often differentiable and the derivatives to any order are obtained by differentiating the power series term by term. Also, power series can be re-expanded around any point in their domain of convergence and the radius of convergence of the new series is equal to the difference between the radius of convergence of the original series and the distance between the original and new centers of the series. Furthermore, it is shown [10] that power series satisfy all the common theorems of real calculus on a closed interval of $\mathcal{R}$, like the intermediate value theorem, the maximum theorem and the mean value theorem. For lack of space, we will only state the results here and refer the reader to [10] for the proofs.

\subsubsection{Differentiability and Re-expandability.}

Theorem 3.13. Let $x_{0} \in \mathcal{R}$ be given, let $\left(a_{n}\right)$ be a sequence in $\mathcal{R}$, let

$$
\lambda_{0}=\limsup _{n \rightarrow \infty}\left\{\frac{-\lambda\left(a_{n}\right)}{n}\right\} \in \mathbb{Q}
$$

and for all $n \geq 0$ let $b_{n}=d^{n \lambda_{0}} a_{n}$. Suppose that the sequence $\left(b_{n}\right)$ is regular; and write $\cup_{n=0}^{\infty} \operatorname{supp}\left(b_{n}\right)=\left\{q_{1}, q_{2}, \ldots\right\}$ with $q_{j_{1}}<q_{j_{2}}$ if $j_{1}<j_{2}$. For all $n \geq 0$, write $b_{n}=\sum_{j=1}^{\infty} b_{n_{j}} d^{q_{j}}$ where $b_{n_{j}}=b_{n}\left[q_{j}\right]$; and let

$$
\eta=\frac{1}{\sup \left\{\limsup _{n \rightarrow \infty}\left|b_{n_{j}}\right|^{1 / n}: j \geq 1\right\}} \text { in } \mathbb{R} \cup\{\infty\}
$$

where $\eta=\infty$ if and only if $\sup \left\{\limsup _{n \rightarrow \infty}\left|b_{n_{j}}\right|^{1 / n}: j \geq 1\right\}=0$. Then, for all $\sigma \in \mathbb{R}$ satisfying $0<\sigma<\eta$, the function $f:\left[x_{0}-\sigma d^{\lambda_{0}}, x_{0}+\sigma d^{\lambda_{0}}\right] \rightarrow \mathcal{R}$, given by $f(x)=\sum_{n=0}^{\infty} a_{n}\left(x-x_{0}\right)^{n}$, is infinitely often differentiable on the interval $\left[x_{0}-\sigma d^{\lambda_{0}}, x_{0}+\sigma d^{\lambda_{0}}\right]$, and the derivatives are given by $f^{(k)}(x)=g_{k}(x)=$ $\sum_{n=k}^{\infty} n(n-1) \cdots(n-k+1) a_{n}\left(x-x_{0}\right)^{n-k}$ for all $x \in\left[x_{0}-\sigma d^{\lambda_{0}}, x_{0}+\sigma d^{\lambda_{0}}\right]$ and 
for all $k \geq 1$. In particular, we have that $a_{k}=f^{(k)}\left(x_{0}\right) / k$ ! for all $k=0,1,2, \ldots$; and hence for all $x \in\left[x_{0}-\sigma d^{\lambda_{0}}, x_{0}+\sigma d^{\lambda_{0}}\right]$, we have that

$$
f(x)=\sum_{n=0}^{\infty} \frac{f^{(n)}\left(x_{0}\right)}{n !}\left(x-x_{0}\right)^{n} .
$$

Theorem 3.14 (Re-expansion of Power Series). Let $x_{0} \in \mathcal{R}$ be given, let $\left(a_{n}\right)$ be a regular sequence in $\mathcal{R}$, with $\lambda_{0}=\lim _{\sup _{n \rightarrow \infty}}\left\{-\lambda\left(a_{n}\right) / n\right\}=0$; and let $\eta \in \mathbb{R}$ be the radius of weak convergence of $f(x)=\sum_{n=0}^{\infty} a_{n}\left(x-x_{0}\right)^{n}$, given by Equation (3.6). Let $y_{0} \in \mathcal{R}$ be such that $\left|\Re\left(y_{0}-x_{0}\right)\right|<\eta$. Then, for all $x \in \mathcal{R}$ satisfying $\left|\Re\left(x-y_{0}\right)\right|<\eta-\left|\Re\left(y_{0}-x_{0}\right)\right|$, we have that $\sum_{k=0}^{\infty} f^{(k)}\left(y_{0}\right) /(k !)\left(x-y_{0}\right)^{k}$ converges weakly to $f(x)$; i.e.

$$
\sum_{k=0}^{\infty} \frac{f^{(k)}\left(y_{0}\right)}{k !}\left(x-y_{0}\right)^{k}=f(x)=\sum_{n=0}^{\infty} a_{n}\left(x-x_{0}\right)^{n} .
$$

Moreover, the radius of weak convergence of $\sum_{k=0}^{\infty} f^{(k)}\left(y_{0}\right) /(k !)\left(x-y_{0}\right)^{k}$ is exactly $\eta-\left|\Re\left(y_{0}-x_{0}\right)\right|$.

3.2.2. Expandable Functions. In this section, we introduce a class of functions on $\mathcal{R}$ that are given locally by power series and for which all the common theorems of real calculus hold.

Definition 3.15. Let $a, b \in \mathcal{R}$ be such that $0<b-a \sim 1$, let $f:[a, b] \rightarrow \mathcal{R}$ and let $x_{0} \in[a, b]$. Then we say that $f$ is expandable at $x_{0}$ if and only if there exists $\delta>0$, finite in $\mathcal{R}$, and there exists a regular sequence $\left(a_{n}\left(x_{0}\right)\right)$ in $\mathcal{R}$ such that, under weak convergence, $f(x)=\sum_{n=0}^{\infty} a_{n}\left(x_{0}\right)\left(x-x_{0}\right)^{n}$ for all $x \in\left(x_{0}-\delta, x_{0}+\delta\right) \cap[a, b]$.

Definition 3.16. Let $a, b \in \mathcal{R}$ be such that $0<b-a \sim 1$ and let $f:[a, b] \rightarrow \mathcal{R}$. Then we say that $f$ is expandable on $[a, b]$ if and only if $f$ is expandable at each $x \in[a, b]$.

Definition 3.17. Let $a<b$ in $\mathcal{R}$ be such that $t=\lambda(b-a) \neq 0$ and let $f$ : $[a, b] \rightarrow \mathcal{R}$. Then we say that $f$ is expandable on $[a, b]$ if and only if the function $g:\left[d^{-t} a, d^{-t} b\right] \rightarrow \mathcal{R}$, given by $g(x)=f\left(d^{t} x\right)$, is expandable on $\left[d^{-t} a, d^{-t} b\right]$.

Lemma 3.18. Let $a<b$ be given in $\mathcal{R}$, let $f, g:[a, b] \rightarrow \mathcal{R}$ be expandable on $[a, b]$ and let $\alpha \in \mathcal{R}$ be given. Then $f+\alpha g$ and $f \cdot g$ are expandable on $[a, b]$.

Theorem 3.19. Let $a<b$ and $c<e$ in $\mathcal{R}$ be given. Let $f:[a, b] \rightarrow \mathcal{R}$ be expandable on $[a, b]$, let $g:[c, e] \rightarrow \mathcal{R}$ be expandable on $[c, e]$, and let $f([a, b]) \subset[c, e]$. Then $g \circ f$ is expandable on $[a, b]$.

Theorem 3.20 (Intermediate Value Theorem). Let $a<b$ in $\mathcal{R}$ be given and let $f:[a, b] \rightarrow \mathcal{R}$ be expandable on $[a, b]$. Then $f$ assumes on $[a, b]$ every intermediate value between $f(a)$ and $f(b)$.

Using Theorem 3.13, we readily obtain the following result.

Theorem 3.21. Let $a<b$ in $\mathcal{R}$ be given, and let $f:[a, b] \rightarrow \mathcal{R}$ be expandable on $[a, b]$. Then $f$ is infinitely often differentiable on $[a, b]$, and for any positive integer $m$, we have that $f^{(m)}$ is expandable on $[a, b]$. Moreover, if $f$ is given locally around $x_{0} \in[a, b]$ by $f(x)=\sum_{n=0}^{\infty} a_{n}\left(x_{0}\right)\left(x-x_{0}\right)^{n}$, then $f^{(m)}$ is given by $f^{(m)}(x)=$ $\sum_{n=m}^{\infty} n(n-1) \cdots(n-m+1) a_{n}\left(x_{0}\right)\left(x-x_{0}\right)^{n-m}$. In particular, we have that $a_{m}\left(x_{0}\right)=f^{(m)}\left(x_{0}\right) / m$ ! for all $m=0,1,2, \ldots$. 
Theorem 3.22. Let $a<b$ in $\mathcal{R}$ be given and let $f:[a, b] \rightarrow \mathcal{R}$ be expandable on $[a, b]$. Then $f$ assumes a maximum and a minimum on $[a, b]$.

Corollary 3.23. Let $a<b$ in $\mathcal{R}$ be given and let $f:[a, b] \rightarrow \mathcal{R}$ be expandable on $[a, b]$. Then there exist $m, M \in \mathcal{R}$ such that $f([a, b])=[m, M]$.

Theorem 3.24 (Rolle's Theorem). Let $a<b$ in $\mathcal{R}$ be given and let $f:[a, b] \rightarrow \mathcal{R}$ be expandable. Suppose $f(a)=f(b)$. Then there exists $c \in(a, b)$ such that $f^{\prime}(c)=0$.

Theorem 3.25 (Mean Value Theorem). Let $a<b$ in $\mathcal{R}$ be given and let $f:[a, b] \rightarrow$ $\mathcal{R}$ be expandable on $[a, b]$. Then there exists $c \in(a, b)$ such that

$$
f^{\prime}(c)=\frac{f(b)-f(a)}{b-a} .
$$

Corollary 3.26. Let $a<b$ in $\mathcal{R}$ be given, and let $f:[a, b] \rightarrow \mathcal{R}$ be expandable on $[a, b]$. Then the following are true.

(i) If $f^{\prime}(x) \neq 0$ for all $x \in(a, b)$ then either $f^{\prime}(x)>0$ for all $x \in(a, b)$ and $f$ is strictly increasing on $[a, b]$, or $f^{\prime}(x)<0$ for all $x \in(a, b)$ and $f$ is strictly decreasing on $[a, b]$.

(ii) If $f^{\prime}(x)=0$ for all $x \in(a, b)$, then $f$ is constant on $[a, b]$.

\section{REFERENCES}

[1] M. Berz. Analytical and Computational Methods for the Levi-Civita Fields. In these proceedings.

[2] M. Berz. Analysis on a nonarchimedean extension of the real numbers. Lecture Notes, 1992 and 1995 Mathematics Summer Graduate Schools of the German National Merit Foundation. MSUCL-933, Department of Physics, Michigan State University, 1994.

[3] M. Berz. Calculus and numerics on Levi-Civita fields. In M. Berz, C. Bischof, G. Corliss, and A. Griewank, editors, Computational Differentiation: Techniques, Applications, and Tools, pages 19-35, Philadelphia, 1996. SIAM.

[4] D. Laugwitz. Tullio Levi-Civita's work on nonarchimedean structures (with an Appendix: Properties of Levi-Civita fields). In Atti Dei Convegni Lincei 8: Convegno Internazionale Celebrativo Del Centenario Della Nascita De Tullio Levi-Civita, Academia Nazionale dei Lincei, Roma, 1975.

[5] Tullio Levi-Civita. Sugli infiniti ed infinitesimi attuali quali elementi analitici. Atti Ist. Veneto di Sc., Lett. ed Art., 7a, 4:1765, 1892

[6] Tullio Levi-Civita. Sui numeri transfiniti. Rend. Acc. Lincei, 5a, 7:91,113, 1898.

[7] L. Neder. Modell einer Leibnizschen Differentialrechnung mit aktual unendlich kleinen Größen. Mathematische Annalen, 118:718-732, 1941-1943.

[8] K. Shamseddine. New Elements of Analysis on the Levi-Civita Field. PhD thesis, Michigan State University, East Lansing, Michigan, USA, 1999. also MSUCL-1147.

[9] K. Shamseddine and M. Berz. Exception Handling in Derivative Computation with Nonarchimedean Calculus. In M. Berz, C. Bischof, G. Corliss, and A. Griewank, editors, Computational Differentiation: Techniques, Applications, and Tools, pages 37-51, Philadelphia, 1996. SIAM.

[10] K. Shamseddine and M. Berz. An Exhaustive Study of Power Series on the Levi-Civita Field. Transactions of the American Mathematical Society, submitted. also MSUCL-1163.

Department of Mathematics and Department of Physics and Astronomy, Michigan State University, East Lansing, MI 48824

E-mail address: shamseddine@nscl.msu.edu

Department of Physics and Astronomy, Michigan State University, East Lansing, Mi 48824

E-mail address: berz@msu.edu 